\title{
The Versatile Type V CRISPR Effectors and Their Application Prospects
}

\author{
Baisong Tong ${ }^{1,2,3 \dagger}$, Huina Dong ${ }^{2,3 \dagger}$, Yali Cui ${ }^{2,3}$, Pingtao Jiang ${ }^{1,2,3}$, Zhaoxia Jin ${ }^{1 *}$ and \\ Dawei Zhang ${ }^{2,3,4 *}$
}

${ }^{1}$ School of Biological Engineering, Dalian Polytechnic University, Dalian, China, ${ }^{2}$ Tianjin Institute of Industrial Biotechnology, Chinese Academy of Sciences, Tianjin, China, ${ }^{3}$ Key Laboratory of Systems Microbial Biotechnology, Chinese Academy of Sciences, Tianjin, China, ${ }^{4}$ University of Chinese Academy of Sciences, Beijing, China

OPEN ACCESS

Edited by:

Guei-Sheung Liu,

University of Tasmania, Australia

Reviewed by:

Hetian Lei,

Shenzhen Eye Hospital, China Yu Fan Chuang,

University of Tasmania, Australia

*Correspondence:

Zhaoxia Jin

jinzx2018@163.com

Dawei Zhang

zhang_dw@tib.cas.cn

†These authors have contributed equally to this work

Specialty section

This article was submitted to

Cellular Biochemistry,

a section of the journal

Frontiers in Cell and Developmental

Biology

Received: 27 October 2020 Accepted: 21 December 2020

Published: 04 February 2021

Citation:

Tong B, Dong H, Cui Y, Jiang P, Jin Z and Zhang $D$ (2021) The Versatile Type V CRISPR Effectors and Their

Application Prospects.

Front. Cell Dev. Biol. 8:622103.

doi: 10.3389/fcell.2020.622103
The class II clustered regularly interspaced short palindromic repeats (CRISPR)-Cas systems, characterized by a single effector protein, can be further subdivided into types II, V, and VI. The application of the type II CRISPR effector protein Cas9 as a sequence-specific nuclease in gene editing has revolutionized this field. Similarly, Cas13 as the effector protein of type $\mathrm{VI}$ provides a convenient tool for RNA manipulation. Additionally, the type $\mathrm{V}$ CRISPR-Cas system is another valuable resource with many subtypes and diverse functions. In this review, we summarize all the subtypes of the type $\checkmark$ family that have been identified so far. According to the functions currently displayed by the type $\vee$ family, we attempt to introduce the functional principle, current application status, and development prospects in biotechnology for all major members.

Keywords: CRISPR-Cas systems, type V effectors, genome editing, nucleic acid detection platforms, transcriptional regulation, base editor

\section{INTRODUCTION}

The CRISPR (clustered regularly interspaced short palindromic repeats)-Cas (CRISPR-associated protein) system is an acquired immune mechanism, mostly found in bacteria and archaea as a defense against environmental mobile genetic elements (MGEs), such as phages, plasmids, and transposons (Sorek et al., 2013; Koonin et al., 2017). The CRISPR system has been divided into class I and class II according to the current classification method, and their effector nuclease modules are composed of multiple subunits or single proteins, respectively (Makarova et al., 2015). Class II is further divided into type II and type V with DNA interference activity, as well as type VI with RNA interference activity (Hajizadeh Dastjerdi et al., 2019).

Like other CRISPR-Cas systems, the type V system consists of three parts, i.e., the effector protein module, the acquisition module, and the CRISPR array (Sorek et al., 2013). When MGEs from the environment invade the host cell, Cas1 and Cas2 in the acquisition module form a complex to intercept a short segment of the invading genetic information named a protospacer, next to a short motif called the protospacer-adjacent motif (PAM), and insert it into a chain of repeated sequences in the CRISPR array (Yosef et al., 2012; Nunez et al., 2015). The CRISPR array is then transcribed to produce pre-CRISPR RNA (pre-crRNA), which is further processed by RNase III or effector proteins in its own CRISPR system to form mature CRISPR RNA (crRNA). Some CRISPR systems also transcribe a trans-activating crRNA (tracrRNA) that is mostly complementary to the repeat sequences in the CRISPR array. Mature crRNA is fused with tracrRNA, or crRNA alone acts as a guide RNA (gRNA) and combines with the effector nuclease in the effector module, which then matches the invading sequence and exerts targeted cleavage activity (Murugan et al., 2017). 
The effector proteins of the type $\mathrm{V}$ family are diverse at the $\mathrm{N}$-terminus but retain a unified RuvC-like endonuclease (RuvC) domain at the C-terminus, derived from the TnpB protein encoded by autonomous or non-autonomous transposons (Shmakov et al., 2017). According to the dissimilar CRISPR effectors, the type $\mathrm{V}$ system is further subdivided into many subtypes, including types $\mathrm{V}$-A to $\mathrm{V}-\mathrm{I}$, type $\mathrm{V}-\mathrm{K}$, type $\mathrm{V}-\mathrm{U}$, and CRISPR-Cas $\Phi$ (Hajizadeh Dastjerdi et al., 2019; Pausch et al., 2020). In these subtype systems, the corresponding effector proteins have shown a variety of functions, whereby some can act not only on double-stranded DNA (dsDNA) but also on singlestranded DNA (ssDNA) and single-stranded RNA (ssRNA). This multifunctionality has put the type V CRISPR-Cas system into the focus of recent studies. However, there is still no review that systematically summarizes these various type V CRISPR systems. The overall sorting is conducive to the application of type $\mathrm{V}$ systems and a profound understanding of CRISPR systems, which we wish to promote with this review.

\section{HISTORY OF THE TYPE V FAMILY}

Type V-A, which was identified as the first known type $\mathrm{V}$ system in the human pathogenic bacterium Francisella tularensis in 2013, has the signature effector protein Cas12a (formerly designated Cpf1) (Schunder et al., 2013). Directed by gRNA, Cas12a successively cleaves the non-complementary and complementary strands of the targeted DNA segment using a single RuvC nuclease domain (Jeon et al., 2018). This is significantly different from the type II CRISPR enzyme Cas9, which uses the HNH-nuclease domain and RuvC-like nuclease domain to cut the two strands of the targeted DNA segment, respectively (Sternberg et al., 2015). The C-terminus with only one RuvC endonuclease domain is the defining characteristic of the type V CRISPR system (Makarova et al., 2015).

In order to explore more unknown type V systems, researchers designed a sophisticated in silico analysis approach using the Cas1 protein, which is widespread and highly conserved among the CRISPR-Cas systems, as the "seed" to query the sequence information in the available terabase-scale database. Through this method, Shmakov et al. identified type V-B and type VC in 2015 (Shmakov et al., 2015), Harrington et al. identified type V-F in 2018 (Harrington et al., 2018), and Yan et al. identified type V-G, type V-H, and type V-I in 2019 (Yan et al., 2019). By querying uncultivated bacteria in groundwater and sediment samples via genome-resolved metagenomics, Burstein and colleagues identified type V-D and type V-E in 2017 (Burstein et al., 2017).

The other way to identify CRISPR-Cas systems, including type V-U systems and the CRISPR-Cas $\Phi$ system, is using CRISPR arrays with a fixed structural pattern as the "anchor." There is no acquisition module near these CRISPR locus, which indicates that these systems cannot be identified by Cas 1 and also indicates that there is a novel mechanism different from Cas 1 and Cas2 in the adaptation phase of these CRISPR systems. Shmakov et al. discovered a series of novel type $\mathrm{V}$-U systems, including $\mathrm{V}$ U1, V-U2, V-U3, V-U4, and V-U5 (Shmakov et al., 2017). The spacer in the CRISPR array is consistent with some phage motifs, providing evidences for the activity of this CRISPR locus. In another investigation, researchers were surprised to find that the type V-U5 system is closely associated to a Tn7-like transposon (Faure et al., 2019). This unusual characteristic makes type V-U5 different from type $\mathrm{V}-\mathrm{U}$, and it was reclassified as the independent subtype V-K. In huge phages existing in diverse ecosystems, AlShayeb et al. identified type II, type V-I, type V-U, and type V-F, which are never found in phages, and the CRISPR-Cas $\Phi$ system, which is not reported in bacteria and archaea. In these systems, their spacers target the host chromosome or other competitive phages, which facilitate phage invasion (Al-Shayeb et al., 2020).

As described in the Introduction section, these different type $\mathrm{V}$ subtypes can interact with three different substrate types (dsDNA, ssDNA, and ssRNA). In addition, different subtypes also exhibit different characteristics with the same substrate (Table 1). In the following section, we classify the subtypes according to different target substrates and discuss the application potential of various type $\mathrm{V}$ subtypes (Figure 1).

\section{TARGETING DSDNA BY THE TYPE V FAMILY}

To date, most of the CRISPR systems identified as belonging to the type $\mathrm{V}$ family have demonstrated targeted RNA-guided dsDNA cleavage activity. Cas12a (type V-A), Cas12b (type V$\mathrm{B}$ ), and Cas12e (type V-E) have been studied in detail, and their structural and functional characteristics are relatively clear. After the effector protein binds the gRNA to form a binary complex, it specifically recognizes the 5 ' T-rich PAM and promotes target DNA unwinding, specified by Watson-Crick base pairing with the guide sequence of crRNA (Stella et al., 2017). At the same time, the non-target strand (NTS) of the target sequence is displaced, forming a so-called "R-loop" structure. The RuvC domain cuts the NTS and target strand (TS) successively at PAMdistant sites to form a staggered incision with 5,7 , or 10 nt $5^{\prime}$ overhangs (Yang et al., 2016; Liu J. J. et al., 2019). Notably, this is different from Cas9, which acts on the PAM-proximal bond to produce a blunt end (Jinek et al., 2014). Cas12c, Cas12d, Cas12h, Cas12i, Cas 12k, and Cas12 $\Phi$ also belong to the type V family but are less known. In addition, some of these type $\mathrm{V}$ Cas proteins have dual-nuclease activity, such as Cas12a, Cas12c (Harrington et al., 2020), Cas12i (Yan et al., 2019), and Cas12j (Pausch et al., 2020). They can process pre-crRNA by themselves, which makes them uniquely suitable for multichannel manipulation (Fonfara et al., 2016; Zetsche et al., 2017; Zhang et al., 2017). Although Cas9 can also modify multiple genes at the same time, either multiple separate sgRNA units (including promoter, gRNA, and terminator) or complicated modifications to the CRISPR array are needed, which makes the process cumbersome and inconvenient (Nissim et al., 2014; Sakuma et al., 2014).

\section{Cas12a, Cas12b, and Cas12e Domain Organization and Divergence}

All three proteins have bilobed structures composed of an $\alpha$ helical recognition (REC) lobe and a nuclease (NUC) lobe. 
TABLE 1 | Properties of type $V$ family effectors.

\begin{tabular}{|c|c|c|c|c|c|c|c|c|}
\hline Subtype & $\begin{array}{l}\text { Signature } \\
\text { protein }\end{array}$ & Length (aa) & PAM & $\begin{array}{l}\text { tracrRNA/ } \\
\text { scoutRNA }\end{array}$ & $\begin{array}{l}\text { Processing } \\
\text { Pre-crRNA }\end{array}$ & $\begin{array}{l}\text { Target } \\
\text { substrates }\end{array}$ & Trans-cleavage & References \\
\hline Type V-A & Cas12a & $1,200-1,500$ & $(T) T T V^{a}$ & No & Yes & dsDNA and ssDNA & ssDNA & Zetsche et al., 2015 \\
\hline Type V-B & Cas12b & $\sim 1,300$ & TTN & tracrRNA & No & dsDNA and ssDNA & ssDNA & Shmakov et al., 2015 \\
\hline Type V-C & Cas12c & $1,200-1,300$ & TG or TN & scoutRNA & Yes & dsDNA and ssDNA & ssDNA & $\begin{array}{l}\text { Yan et al., 2019; } \\
\text { Harrington et al., } 2020\end{array}$ \\
\hline Type V-D & Cas12d & $\sim 1,200$ & TA or TG & scoutRNA & Unconfirmed & dsDNA and ssDNA & ssDNA & $\begin{array}{l}\text { Chen L. X. et al., 2019; } \\
\text { Harrington et al., } 2020\end{array}$ \\
\hline Type V-E & Cas12e & $\sim 1,000$ & TTCN & tracrRNA & Unconfirmed & dsDNA & ssDNA & Liu J. J. et al., 2019 \\
\hline Type V-F & Cas14 & $400-700$ & $\mathrm{NO}$ & tracrRNA & No & ssDNA & ssDNA & Harrington et al., 2018 \\
\hline Type V-G & Cas12g & $\sim 800$ & $\mathrm{NO}$ & tracrRNA & No & ssRNA & ssRNA and ssDNA & Yan et al., 2019 \\
\hline Type V-H & Cas12h & $\sim 900$ & $\mathrm{RTR}^{\mathrm{b}}$ & No & No & dsDNA and ssDNA & ssDNA & Yan et al., 2019 \\
\hline Type V-I & Cas12i & $\sim 1,100$ & TTN & No & Yes & dsDNA and ssDNA & ssDNA & Yan et al., 2019 \\
\hline Type V-K & Cas12k & $\sim 650$ & GTN & tracrRNA & No & dsDNA & No & Strecker et al., 2019b \\
\hline CRISPR-Cas $\Phi$ & Cas12j & $\sim 750$ & $\mathrm{TBN}^{\mathrm{C}}$ & No & Yes & dsDNA and ssDNA & ssDNA & Pausch et al., 2020 \\
\hline
\end{tabular}

${ }^{a} V$ represents $A, C$, and $G$.

${ }^{b} R$ represents $A$ and $G$.

${ }^{c} B$ represents $C, G$, and $T$.

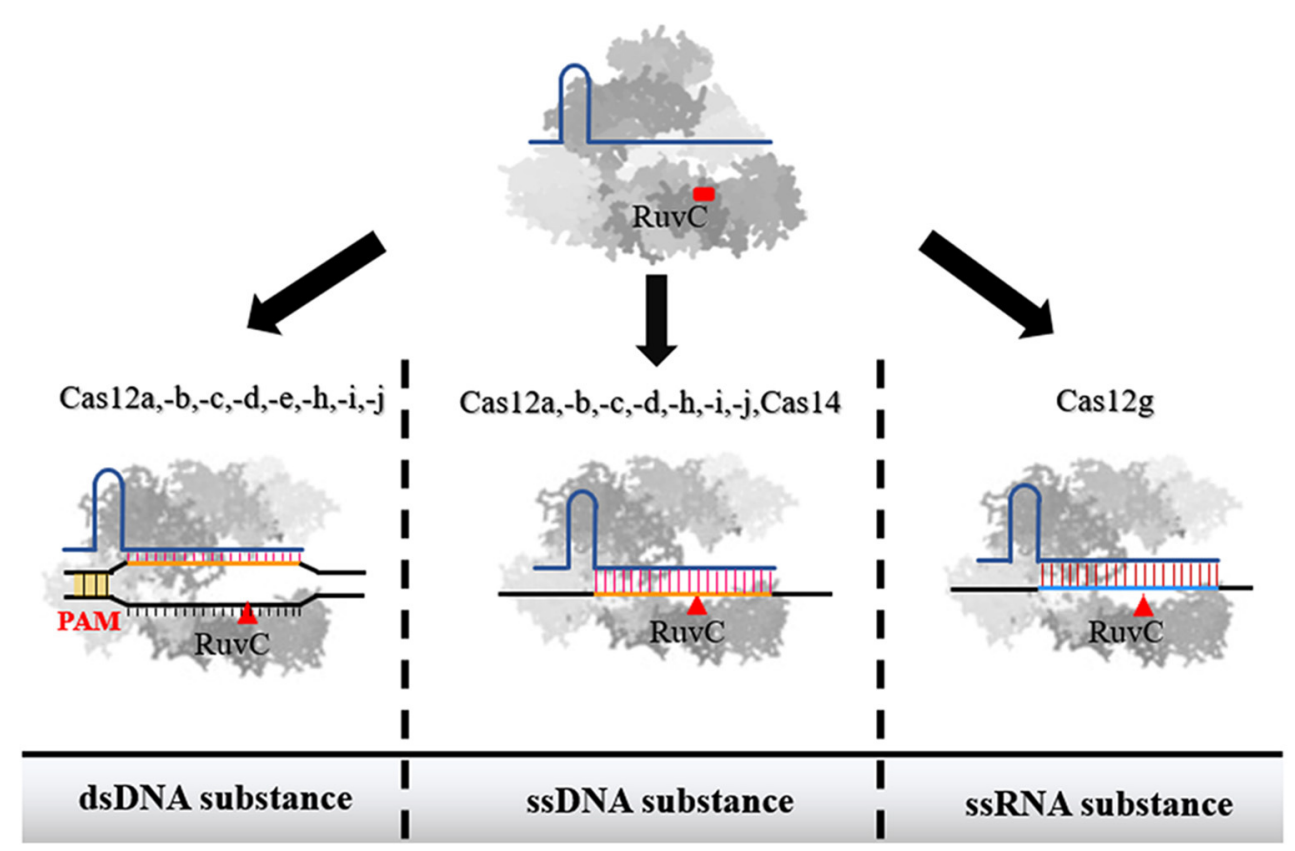

FIGURE 1 | The recognition substance of effector proteins of each subtype in type V family. Among them, Cas12a, Cas12b, Cas12c, Cas12d, Cas12h, Cas12i, and Cas12j were confirmed to have the ability to interact with both dsDNA and ssDNA. Cas14 only has the ability to act on ssDNA. Cas12g can recognize ssRNA. When the single-stranded nucleic acid molecule is used as a substance, the binding of Cas protein is not restricted by PAM.

The two lobes are connected by a bridge helix $(\mathrm{BH})$ motif (Figure 2A). The REC lobe contains two REC domains (REC1 and REC2), which mainly help accommodate and stabilize the crRNA-target DNA hybrid after forming the "R-loop" (Gao et al., 2016). In the NUC lobe, Cas12a contains a RuvC domain, a PAM identical (PI) domain, an oligonucleotide-binding (OB) domain, and a NUC domain. The NUC domain was previously considered to be another nuclease domain of type $\mathrm{V}$ family enzymes, but recently, researchers have proposed to rename it as the TSL (target strand loading) domain to better reflect its actual role (Liu J. J. et al., 2019). The OB domain of Cas12a combines with the secondary structure at the $5^{\prime}$ end of gRNA to form a binary complex, and the initial $5 \mathrm{nt}$ of crRNA in the PAM-proximal have an A-form-like conformation (Dong et al., 2016; Swarts and Jinek, 2019). These several pre-ordered nucleotides are defined as the "seed sequence." When the PI 
domain recognizes the PAM and unzips the targeted dsDNA, the seed sequence immediately interact with the target strands, laying a foundation for the extension of the R-loop (Stella et al., 2017). Consequently, mismatches in the seed sequence region are often devastating for the targeting function of the Cas protein. Cas $12 \mathrm{~b}$ does not have an independent PI domain. After combining with gRNA to form a binary complex, the OB domain and REC-I will form a pre-organized PAM duplex cleft, where helices $\alpha 5$ and $\alpha 6$ and the flexible loop between $\alpha 4$ and $\alpha 5$ in the RECI occupy a similar position to that of the PI domain in Cas12a and identify PAM (Yang et al., 2016; Wu et al., 2017). Cas12e also contains the unique non-target strand binding (NTSB) domain, which is required for target DNA unwinding. This additional domain suggests that the Cas12e-gRNA complex recognizes the target sequence and combines with it to form a ternary complex via a mechanism different from those of other CRISPR enzymes (Liu J. J. et al., 2019).

\section{Other Cas Proteins That Recognize dsDNA}

Some newly discovered Cas proteins from the type V family also have dsDNA cleavage activity, albeit only proven by biochemical experiment, including Cas12c, Cas12d, Cas12h, Cas12i, and Cas12j (Chen L. X. et al., 2019; Yan et al., 2019; Pausch et al., 2020). It is worth noting that although Cas12i induces double-strand breaks (DSB), the efficiency of cutting is different for each strand of the target DNA, with strong cleavage of the non-complementary strands, but very low cleavage efficiency for complementary strands. Consequently, DSB are only produced at very limited frequencies (Yan et al., 2019). This feature makes it possible to use Cas $12 \mathrm{i}$ as a nickase by using a pair of gRNAs to reduce the offtarget rate and achieve large-scale gene fragment manipulation just like nCas9 (Ran et al., 2013a; Standage-Beier et al., 2015).

In addition to targeting dsDNA for cleavage, the type V$\mathrm{K}$ system can target dsDNA for transposition. As mentioned above, the type V-K CRISPR locus is associated with Tn7like transposons (Faure et al., 2019). The range framed by the transposon end sequences (LE and RE) encodes a Cas12k enzyme with a naturally inactivated RuvC domain and the three transposition-related genes $t n s B$, tns $C$, and tniQ (Figure 2B). Previous researches indicated that canonical $\operatorname{Tn} 7$ transposons integrate the cargo gene between LE and RE at a specific Tn7 attachment site $(\operatorname{att} T n 7)$ at higher frequencies or randomly integrate through another mutually exclusive mechanism (Peters, 2014). However, the insertion site of the transposon-encoded CRISPR system is located at a fixed distance downstream of the PAM of the targeting locus. Cas12k, which has lost its DNase activity, does not simply play an RNA-directed recognition role. When Cas12k was replaced with Cas9, there was no transposition phenomenon, indicating that Cas $12 \mathrm{k}$ and the transposase complex have a coupling activation effect (Strecker et al., 2019b). Although Cas12k does not destroy the target site after binding to the target DNA, it has the same targeted immunity effect as the canonical Tn7 transposase in preventing multiple insertions. Consequently, the type V-K system will only insert the cargo gene once in a certain orientation at the same position (Stellwagen and Craig, 1997; Skelding et al., 2003).

\section{Applications Based on dsDNA Recognition Genome Editing}

As an RNA-dependent sequence-specific nuclease, the Cas protein was developed as a gene editing tool at the beginning of research in this field (Ran et al., 2013b). Single or multiple plasmids express effector protein and artificial gRNA, induce

\section{A}

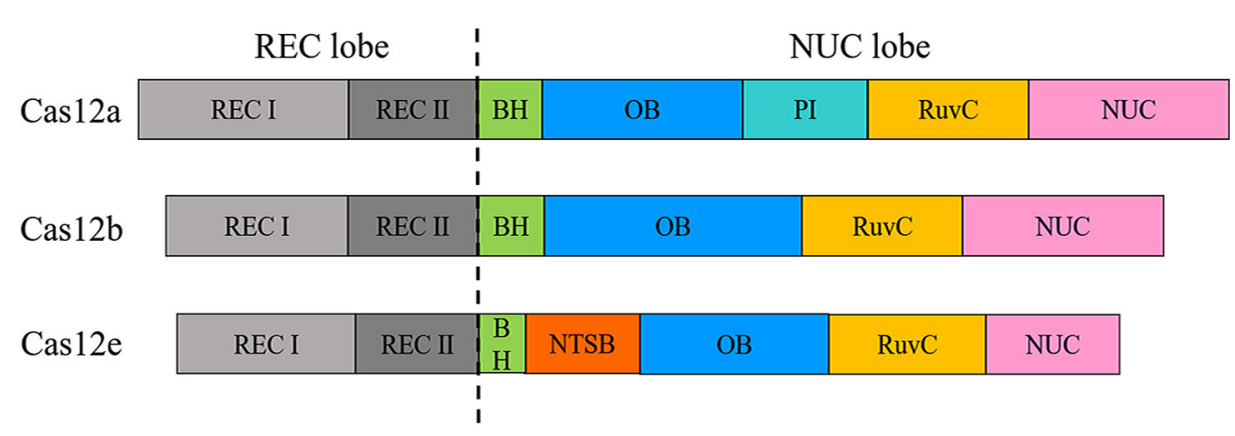

B

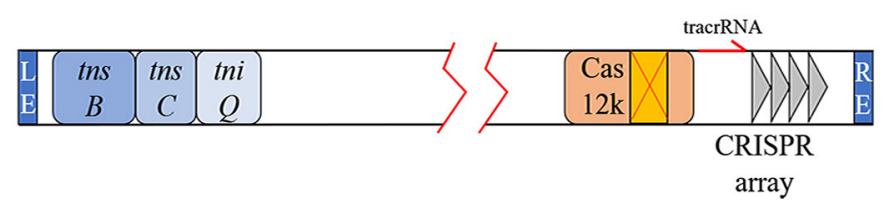

FIGURE 2 | Domain diversity of Cas12a, Cas12b, and Cas12e and type V-K CRISPR loci. (A) The REC lobes of Cas12a, Cas12b, and Cas12e all contain two REC domains. There are some differences in the NUC lobe, including the presence of the PI domain and the additional NTSB domain of Cas12e. (B) Transposon-related genes and CRISPR elements are located at both ends of the transposon. 
DSB in a user-specified manner, and repair the DNA damage through non-homologous end joining (NHEJ) or homologydirected recombination (HDR) (Pawelczak et al., 2018). In the absence of a DNA template, the imprecise NHEJ-based repair will introduce random indels of several nucleotides at the cleavage site. The resulting frameshift mutations usually lead to gene inactivation. In the presence of homologous templates, HDR can achieve gene replacement, insertion, deletion, and other traceless genetic manipulations (Figure 3A). To date, the genetic manipulation toolbox based on Cas12a and Cas $12 \mathrm{~b}$ from the type $\mathrm{V}$ family has been successfully applied in mammals, plants, and bacteria (Table 2).

They, as supplements to Cas9, have been applied in many organisms that are not compatible with Cas9 (Ungerer and Pakrasi, 2016; Jiang et al., 2017). The Cas proteins mentioned above that have only been verified by biochemistry with dsDNAtargeted cleavage capabilities also have the ability to act as alternatives. These type $\mathrm{V}$ Cas proteins recognize a completely different PAM from Cas9, extending the targeting range, and the sticky ends generated at the PAM-distal position contribute to the efficiency of HDR repair (Fagerlund et al., 2015; MorenoMateos et al., 2017). Furthermore, compared with Cas9, Cas12a shows a lower frequency of off-target events (Kim D. et al., 2016; McMahon and Rahdar, 2021).

The CRISPR-Cas system is more convenient to operate in gene editing compared with the previous two generations of targeted gene editing technology, zinc-finger nucleases (ZFN), and transcription activator-like effector nucleases (TALENs), but it is not without its own limitations. The DSB induced by the sequence-specific nucleases at the target sites are fatal in organisms without effective NHEJ or HDR (Xu et al., 2015). To overcome this limitation, researchers attempted to connect different transposases to catalytically inactivated dCas9 (sitespecific mutations were introduced into the $\mathrm{HNH}$ and RuvC domains to abrogate the cleavage activity without affecting the targeted binding effect) to obtain a series of dCas9-transposase fusion proteins [such as dCas9-Himarl (Chen and Wang, 2019), dCas9-piggyBac (Hew et al., 2019), and dCas9-Sleeping Beauty (Kovač et al., 2020)]. These fusions limit the random insertion of transposase, so that transposition occurs only near the point where dCas9 is targeted. Although the transposition reaction does not depend on the DSB repair ability of the host, limitations of the fusion protein by the PAM of dCas9 and the transposase insertion hot spot make this tool less flexible. For example, dCas9-Himar1 requires a TA dinucleotide within 15 bp behind the $5^{\prime}$ end of the gRNA (Chen and Wang, 2019).

The insertion site of the type V-K CRISPR system is only related to the target position of the gRNA, and there is no hot spot preference (Strecker et al., 2019b). This makes the operable range of this system more extensive, and the type V-K system has already been successfully used as a programmable gene editing tool in Escherichia coli. Through biochemical experiments, it was found that the Cas $12 \mathrm{k}$ enzyme of this transposon-associated type V CRISPR system preferentially recognizes the $5^{\prime}$-GTN PAM. Cargo genes are inserted unidirectionally at positions 60-66 downstream of the PAM, and the insertion efficiency of a 2$\mathrm{kb}$ cargo gene can be up to $80 \%$ without antibiotic screening. Although the type V-K system that can integrate exogenous

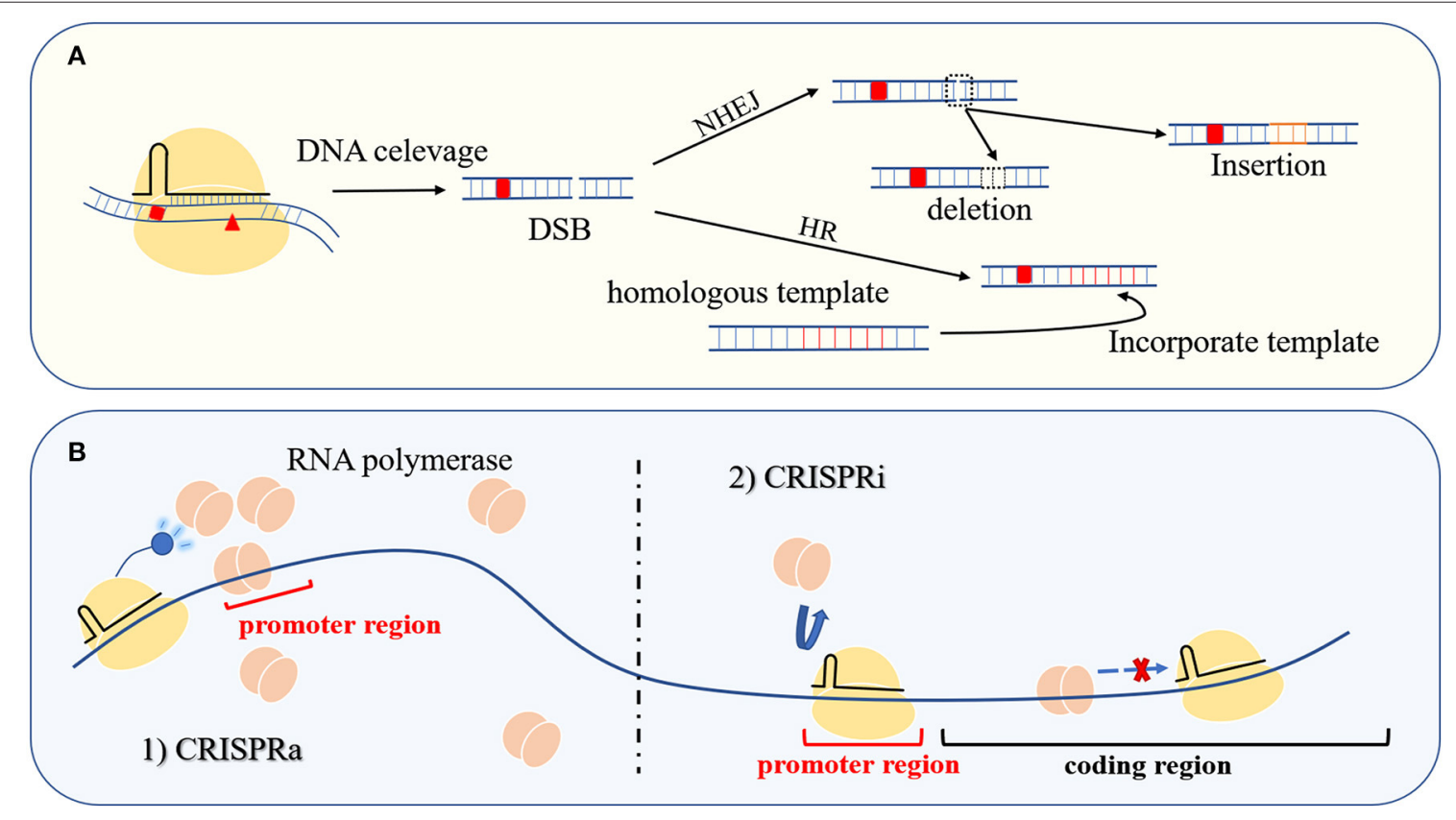

FIGURE 3 | Gene editing and transcriptional regulation. (A) The Cas protein at the user-specified site causes DSB to achieve seamless operation at the targeted location through NHEJ or HDR. (B) The left side of the picture is CRISPR-mediated transcription activation (CRISPRa), and the right side is CRISPR-mediated transcription repression (CRISPRi). Control gene transcription is through gain and loss of RNA polymerase, respectively. 
TABLE 2 | Summary of organisms whose genomes were successfully edited using Cas 12a and Cas12b.

\begin{tabular}{|c|c|c|c|}
\hline \multicolumn{2}{|c|}{ Bacteria } & \multirow{2}{*}{$\begin{array}{l}\text { Animal } \\
\text { - Human cell (Strecker et al., 2019a; } \\
\text { Teng et al., 2019b) } \\
\text { - Zebrafish (Fernandez et al., 2018) } \\
\text { - Mice (Kim Y. et al., 2016; Teng } \\
\text { et al., 2018) } \\
\text { - Pig (Zou et al., 2019) } \\
\text { - Drosophila (Port and Bullock, 2016) }\end{array}$} & \multirow[b]{2}{*}{ 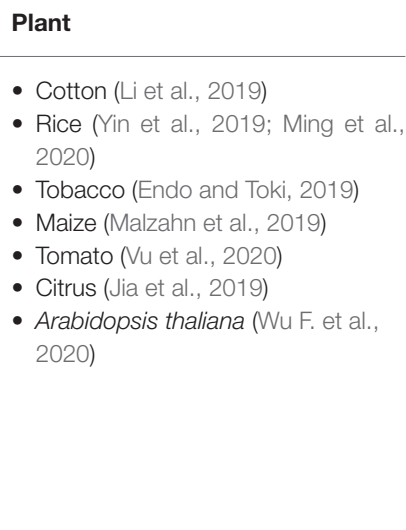 } \\
\hline $\begin{array}{l}\text { - Saccharomyces cerevisiae (Li Z. H. } \\
\text { et al., 2018) } \\
\text { - Yarrowia lipolytica (Yang et al., } \\
\text { 2020) } \\
\text { - Ashbya gossypii (Jiménez et al., } \\
\text { 2020) } \\
\text { - Aspergillus nidulans (Vanegas et al., } \\
\text { 2019) } \\
\text { - Clostridium beijerinckii (Zhang J. } \\
\text { et al., 2018) } \\
\text { - Clostridium difficile (Hong et al., } \\
\text { 2018) } \\
\text { - Corynebacterium glutamicum } \\
\text { (Zhang et al., 2019) }\end{array}$ & $\begin{array}{l}\text { - Zymomonas mobilis (Shen et al., } \\
\text { 2019) } \\
\text { - Pseudomonas putida (Sun J. et al., } \\
\text { 2018) } \\
\text { - Streptomyces coelicolor (Li L. } \\
\text { et al., 2018) } \\
\text { - Mycobacterium smegmatis (Sun B. } \\
\text { et al., 2018) } \\
\text { - Bacillus subtilis (Wu Y. et al., 2020) } \\
\text { - Cyanobacterium Anabaena (Niu } \\
\text { et al., 2019) } \\
\text { - Chlamydomonas reinhardtii } \\
\text { (Ferenczi et al., 2017) }\end{array}$ & & \\
\hline
\end{tabular}

genes into a certain position relies on a self-sufficient mechanism, both the LE/RE and the 5-bp repeat sequence generated by transposition are inevitably inserted together, which leads to redundancy in the genome (Strecker et al., 2019b). This is a major obstacle to the widespread application of type $\mathrm{V}-\mathrm{K}$ and the focus of future optimization.

\section{Transcriptional Regulation}

Mutation of active site residues in the RuvC nuclease domain of the Cas nucleases in the type $\mathrm{V}$ family can be used to produce deactivated Cas proteins (dCas). Guided by gRNA, the dCas protein only binds to the target site and does not exhibit nuclease activity. When dCas targets the promoter region or coding region of a structural gene, it inhibits gene transcription by competing with RNA polymerase for promoter binding or by blocking polymerase migration. The resulting gene repression method is known as CRISPR interference (CRISPRi) (Figure 3B) (Kim et al., 2017; Zhang et al., 2017). In addition, dCas proteins can be used as targeting moieties for delivering a functional protein to a specific loci, including transcription factors, transcriptional activators, and RNA polymerase subunits. When the fusion protein is targeted to the proper distance from the gene transcription start site (TSS), the gene expression can be improved through the interaction between these functional proteins and RNA polymerase. This transcriptional activation tool based on the CRISPR-Cas system is called CRISPRa (Figure 3B). The emergence of CRISPRi and CRISPRa provides convenient tools for the characterization of gene functions and metabolic regulation (Liu W. et al., 2019; Martella et al., 2019).

As mentioned above, the RNase activity of Cas12a can process a customized CRISPR array. Zhang et al. constructed the CRISPR array to target four locus, and dCas12a successfully inhibited multiple genes simultaneously. Moreover, the efficiency of simultaneously repressing four genes was similar to that of repressing the separated gene (Zhang et al., 2017). Zhang J. L. et al. (2018) used three crRNAs to coordinately target the same gene, which increased the repression efficiency by 6.7 -fold. In addition, Cas12a has been developed into multipurposed tools. For example, Adithya et al. found that when the crRNA length is $<16 \mathrm{bp}$, Cas12a can bind to the target site without cleavage.
On this basis, the gene editing and CRISPRi can be realized simultaneously by transferring crRNAs with different lengths in series (Ramesh et al., 2020). Wu et al. achieved repression and activation on multiple genes simultaneously. When dCas12aRemA (a kind of transcription factor) bound to the crRNA targeted into genes, it successfully downregulated the genes. When dCas12a-RemA bound to the crRNA targeted upstream of the TSS, it successfully upregulated the expression of the gene (Wu Y. et al., 2020).

\section{Base Editing}

The application of CRISPR technology has led to tremendous progress in genetic engineering, but the precise editing of single nucleotides is still a challenge. Aiming to achieve this goal, the rat cytosine deaminase APOBEC and uracil DNA glycosylase (UDG) inhibitor (UGI) were, respectively, fused to the $\mathrm{N}$ - and C-termini of dCas12a. After dCas12a binds to the target site, the R-loop is formed, and the non-complementary ssDNA strand provides the catalytic substrate for APOBEC. Consequently, the cytosine (C) at the 8-13 nt position downstream of the PAM was deaminated to uracil (U), and the previous $\mathrm{C}-\mathrm{G}$ base pair was successfully changed to an A-T base pair after DNA replication. At the same time, the C-terminal UGI can inhibit base excision repair by UDG and increase the mutation rate (Figure 4A) (Banno et al., 2018). The same strategy was applied to dCas9, but researchers found that dCas12a-mediated BE had higher editing efficiency and lower off-target rate (Li X. et al., 2018). Although it can improve the efficiency of BE to replace dCas9 with nCas9, nickase activity of nCas9 leads to DNA damage response (DDR) (Wang et al., 2020a). The targeted chromosomal single-base mutations made feasible by this CRISPR-based base editor have great application value in protein engineering and gene therapy.

\section{TARGETING SSDNA BY THE TYPE V FAMILY}

\section{Specific ssDNA Cleavage Activity}

Many of the dsDNA-targeting CRISPR effector proteins belonging to class II have shown concurrent ssDNA-targeting cleavage in vivo and in vitro (Table 1) (Ma et al., 2015). This 

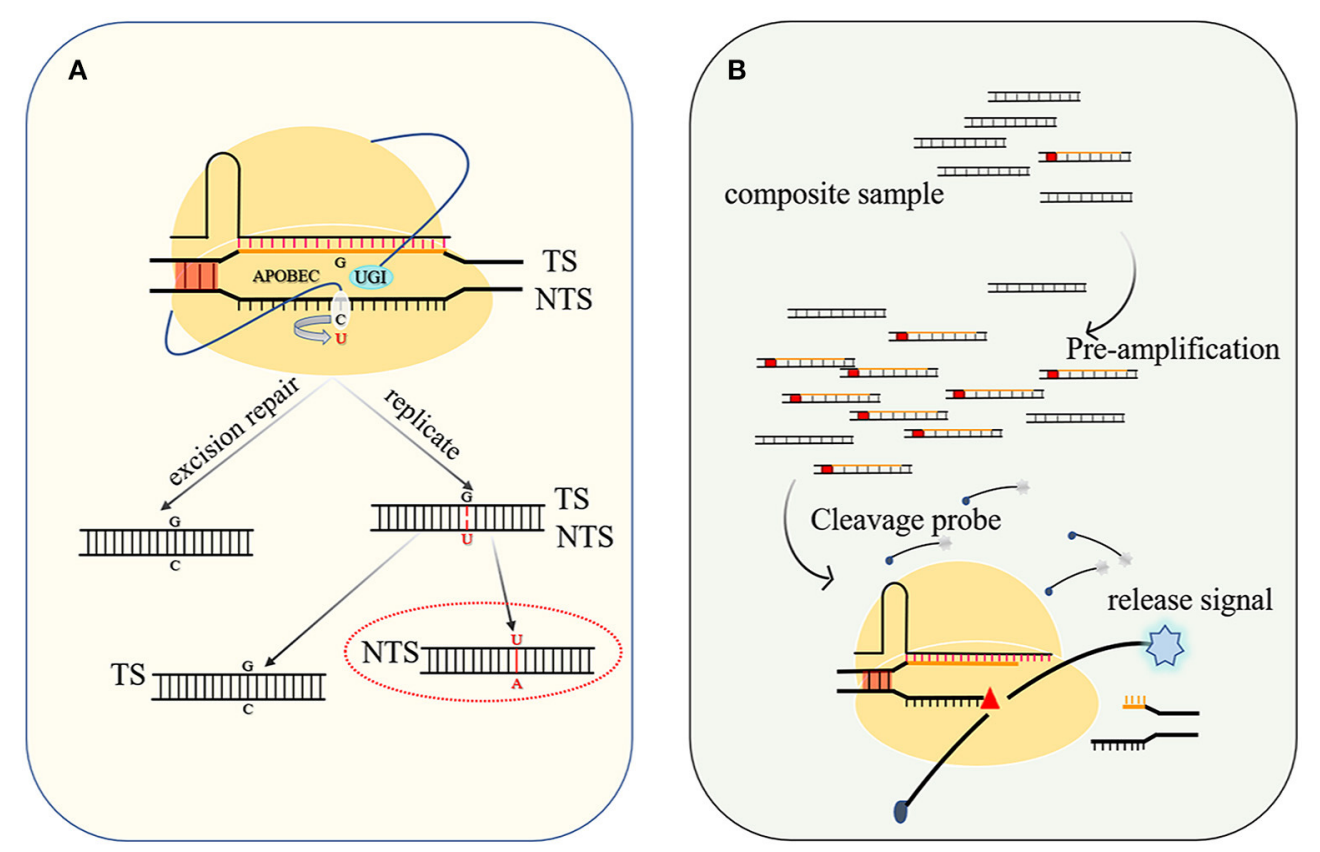

FIGURE 4 | Base editing diagram and principle of nucleic acid detection. (A) When UGI is used to inhibit host base excision repair, APOBEC catalyzes the conversion of $\mathrm{C}$ to $\mathrm{U}$ with higher efficiency, and after DNA replication, a $\mathrm{G}-\mathrm{C}$ base pair is changed to an $\mathrm{A}-\mathrm{U}(\mathrm{T})$ base pair. (B) The targeted nucleic acid undergoes

preamplification to increase its concentration and is cleaved by the Cas protein to activate the trans-cleavage activity of the Cas protein and decomposes the probe to release the detection signal.

ssDNA cleavage activity is not limited by the PAM. As long as the target substrate is complementary to the guide sequence of the crRNA, it will work. The recently identified type V-F system almost only occurs in a group of archaea characterized by small cell and genome sizes, and the signature Cas 14 protein of this system is also very compact, about $400-700$ amino acids, which is half the size of the effector proteins in other type $\mathrm{V}$ systems. The mature tracrRNA and crRNA were obtained through environmental meta-transcriptomic sequence analysis. When these genes were expressed in a heterologous host, although Cas14 could successfully bind the gRNA to form a binary complex, no catalytic activity was observed with either single- or double-stranded DNA or RNA in vivo. However, when the purified Cas14-sgRNA binary complex was incubated with complementary ssDNA in vitro, cleavage activity was observed (Harrington et al., 2018; Savage, 2019). Although no in vivo Cas14 catalytic activity has been detected for the time being, it may be due to the lack of some key factors contained in the natural host of Cas14, which is worthy of further exploration. It also implies that targeted ssDNA cleavage is a widespread and possibly ancestral function.

\section{Non-specific ssDNA Cleavage Activity}

Type $\mathrm{V}$ family proteins have unique non-specific ssDNA cleavage activity (Table 1). After the effector protein cleaves the targeted dsDNA substance, the PAM-distal product is released, but the Cas protein is still bound to the PAM-proximal strand. At this point, the RuvC domain can still access ssDNA substrates and perform non-specific cleavage, which is also known as the promiscuous cleavage of collateral ssDNA or trans-cleavage ( $\mathrm{Li}$ et al., 2018a; Liu J. J. et al., 2019; Swarts and Jinek, 2019).

\section{Applications Based on ssDNA Cleavage Activity}

\section{Nucleic Acid Detection Platform}

The trans-acting DNase activity induced by DNA-specific recognition has been developed into a DNA detection platform by employing a quenched fluorescent ssDNA as the probe. When a cognate target is present in the system, Cas12a severs the targeted entities to activate trans-cleavage activity, thereby also cleaving the surrounding probes to release a detectable fluorescent molecule (Figure 4B). Preamplification of the target sequence by isothermal recombinase-polymerase amplification (RPA) or loop-mediated isothermal amplification (LAMP) can improve the sensitivity, and the detection limit of the desired DNA or RNA can be as low as 10 aM (attomolar) (Li et al., 2018b). After continuously optimizing this detection system and improving the specificity of recognition, the system has been widely used in the discrimination of single nucleotide polymorphisms (Teng et al., 2019a) and genotyping (Harrington et al., 2018) and virus detection, such as severe acute respiratory syndrome coronavirus 2 (SARS-CoV-2) (Broughton et al., 2020; Wang et al., 2020b).

\section{Small Molecule Detection Platform}

Liang et al. combined the sensitivity of allosteric transcription factors (aTFs) for small molecules with the non-specific ssDNA 
cleavage activity of Cas12a to develop a platform for detecting small molecules, named CaT-SMelor (CRISPR-Cas12a and aTFmediated small molecule detector). It is well-known that aTFs have an effector-binding domain and a DNA-binding domain. Following preassembly of the well-designed dsDNA that can be recognized by Cas12a with aTF, a targeted small molecule can bind to the aTF, which then releases the dsDNA. Cas12a in turn binds to the released dsDNA and activates the transactivity to cut the nearby quenched fluorescently labeled ssDNA and to generate a fluorescent detection signal. This system can successfully differentiate nanomolar levels of target molecules from a structurally similar analog (Liang et al., 2019).

\section{TARGETING SSRNA BY THE TYPE V FAMILY}

Type V-G is a recently identified CRISPR system with a small signature protein, Cas12g, which has an intact RuvClike endonuclease domain at the C-terminus (Yan et al., 2019). However, no ssDNA or dsDNA cleavage activity was detected either in vivo or in vitro when Cas $12 \mathrm{~g}$ was incubated with crRNA and tracrRNA. Surprisingly, when the substrates were replaced with cognate ssRNA, Cas12 exhibited targeted cleavage activity without constraint of a PAM sequence. Moreover, after binding to the targeted RNA, the trans-cleavage activity was activated to non-specifically cleave the surrounding ssRNA. Inactivation of the RuvC domain determined that Cas12g's RNA cleavage activity depends on an intact RuvC domain, disproving the previous theory that the RuvC domain could only act on DNA substrates. Although the effector protein Cas13 of the type VI CRISPR system also has RNA-targeting cleavage activity, it cuts RNA via two HEPN domains (Liu et al., 2017).

\section{Applications Based on ssRNA Recognition}

Cas12g has similar functions as Cas13, including RNA-guided RNA-targeting and trans-cleavage activity. With the further study of Cas13, a variety of RNA-specific tools have been developed. Cas12g also has the full corresponding development potential, and its size is only 700 aa. Moreover, its origin from a hot spring metagenome gives it high thermal stability.

\section{RNA-Based Nucleic Acid Detection Platform}

Gootenberg et al. developed a nucleic acid detection platform based on the type VI CRISPR enzyme Cas13. The system is based on preamplifying DNA or RNA samples through RPA or RT-RPA, T7 RNA polymerase transcription of amplified DNA to RNA, and detection by Cas13 via collateral cleavage of an ssRNA probe (Gootenberg et al., 2017). Although Cas13 and Cas12a can only use RNA and DNA as recognition matrices, respectively, because of the amplification steps and the flexible use of transcription and reverse transcription, these two detection platforms can detect samples whose initial substrates are either DNA or RNA. Therefore, when the sample cannot be amplified and transformed or the detection purpose does not allow the preamplification step, the detection of DNA or RNA requires the division of the two platforms. For example, Chen et al. used a nucleic acid detection platform to monitor the degree of RNA methylation. Because methylated nucleotides are not complementary to crRNA, the intensity of the fluorescent signal reflects the methylation degree of RNA (Chen Y. et al., 2019). For this purpose, the Cas13-based RNA nucleic acid detection platform is the only option available to date. If Cas12g instead of Cas13 is developed as a portable in vitro RNA detection kit, thermal stability is a potential advantage (Chen et al., 2020).

\section{RNA Single-Base Editing}

Adenosine deaminase acting on RNA type 2 (ADAR2) has an $\mathrm{N}$-terminal dsRNA-binding domain and a C-terminal catalytic deamination domain, which can be combined with dsRNA to achieve adenosine-to-inosine transformation through hydrolytic deamination. Furthermore, the ADAR2 deaminase domain (ASAR2 ${ }_{\mathrm{DD}}$ ) can function independently without other cofactors. Cox et al. tethered the hyperactivating mutant $\mathrm{ADAR} 2_{\mathrm{DD}}$ to the catalytically inactive Cas13b (dCas13b) and designed the gRNA to introduce A:C mismatches at the desired site (ADAR2 characteristics, higher catalytic activity for A:C mismatch sites on dsRNA) to achieve precise editing of A to I in mammals (Cox et al., 2017). Through multiple rounds of evolution of ADAR2 2 , the team obtained a cytosine deaminase with the ability to convert cytosine to uracil and fused this evolved cytidine deaminase to dCas13 to develop a programmable RNA editing tool for specific $\mathrm{C}$ to $\mathrm{U}$ exchange (Abudayyeh et al., 2019; Aquino-Jarquin, 2020). Cas13b has been shown to be free of PFS (protospacer-flanking site, parallel to PAM in class II) restrictions in mammalian cells, compared with DNA base editors where the target site is restricted to PAM. The RNA base editor based on Cas13 is more flexible and expands the target range in gene therapy, but still faces challenges in viral delivery. Not only does Cas12g have no PAM restrictions, but minimizing the system by using Cas12g is a potential optimization opportunity.

\section{CONCLUSION AND OUTLOOK}

Among the diverse CRISPR-Cas systems, class 2 has the most extensive applications because of having only one effector protein. The type V CRISPR-Cas system has become a shining star due to the growing type $\mathrm{V}$ family and the diversity of its functions. Nevertheless, only the earliest Cas12a and Cas12b have been used in practical applications. In this review, we summarize all the type $\mathrm{V}$ family systems discovered so far and discuss their characteristics in a taxonomic manner. Some of these newly discovered type $\mathrm{V}$ family Cas proteins can be used as candidates in the novel application strategies that are currently being developed. Some of their unique properties such as high mismatch sensitivity, small size, and thermal stability have the potential to further optimize these tools.

Except for Cas12a, Cas12b, and Cas12e, the N-terminal domain and crystal structures of other type $\mathrm{V}$ family Cas proteins have not been resolved. Through the comparison of Cas12a, Cas12b, and Cas12e, it is not difficult to realize that the diversity of the $\mathrm{N}$-terminal domain of the Cas protein reflects the differences in the mechanism of action and the cleavage effect. 
Future analysis of the structure of these Cas proteins will further reveal the relationship between structure and function, which will contribute to the further development and optimization of the CRISPR-Cas system.

In addition to type $\mathrm{V}$-U5, the functions of several other subtypes of the type V-U family (types V-U1, V-U2, V-U3, V-U4) are unknown. Behind the unusual may be born with surprise. In addition, the effector proteins in these families are very similar to TnpB, so further research on the type $\mathrm{V}$-U family will help explain how CRISPR-Cas effectors evolved from TnpB.

\section{AUTHOR CONTRIBUTIONS}

BT wrote the manuscript and performed all of the necessary literature searches. HD, YC, and PJ performed the necessary literature searches. ZJ revised the manuscript and gave valuable

\section{REFERENCES}

Abudayyeh, O. O., Gootenberg, J. S., Franklin, B., Koob, J., Kellner, M. J., Ladha, A., et al. (2019). A cytosine deaminase for programmable single-base RNA editing. Science 365, 382-386. doi: 10.1126/science. aax7063

Al-Shayeb, B., Sachdeva, R., Chen, L. X., Ward, F., Munk, P., Devoto, A., et al. (2020). Clades of huge phages from across earth's ecosystems. Nature 578, 425-431. doi: 10.1038/s41586-020-2007-4

Aquino-Jarquin, G. (2020). Novel engineered programmable systems for ADAR-mediated RNA editing. Mol. Ther. Nucleic Acids 19, 1065-1072. doi: 10.1016/j.omtn.2019.12.042

Banno, S., Nishida, K., Arazoe, T., Mitsunobu, H., and Kondo, A. (2018). Deaminase-mediated multiplex genome editing in Escherichia coli. Nat. Microbiol. 3, 423-429. doi: 10.1038/s41564-017-0102-6

Broughton, J. P., Deng, X., Yu, G., Fasching, C. L., Servellita, V., Singh, J., et al. (2020). CRISPR-Cas12-based detection of SARS-CoV-2. Nat. Biotechnol. 38, 870-874. doi: 10.1038/s41587-020-0513-4

Burstein, D., Harrington, L. B., Strutt, S. C., Probst, A. J., Anantharaman, K., Thomas, B. C., et al. (2017). New CRISPR-Cas systems from uncultivated microbes. Nature 542, 237-241. doi: 10.1038/nature 21059

Chen, L. X., Al-Shayeb, B., Meheust, R., Li, W. J., Doudna, J. A., and Banfield, J. F. (2019). Candidate phyla radiation roizmanbacteria from hot springs have novel and unexpectedly abundant CRISPR-Cas systems. Front. Microbiol. 10:928. doi: $10.3389 /$ fmicb. 2019.00928

Chen, Q. L. R., Lv, P., Li, H., Huang, D., He, P., and Zhang, Y. (2020). Rapid detection of specific nucleic acid fragment nano-fluorescence trace based on CRISPR-Cas12g comprises e.g. using specific nucleic acid fragments in biological samples as research object to prepare nucleic acid targets, and quenching. Chinese Patent NO CN111378722-A. Beijing: China National intellectual property administration.

Chen, S. P., and Wang, H. H. (2019). An engineered Cas-transposon system for programmable and site-directed DNA transpositions. CRISPR J. 2, 376-394. doi: $10.1089 /$ crispr.2019.0030

Chen, Y., Yang, S., Peng, S., Li, W., Wu, F., Yao, Q., et al. (2019). N1-methyladenosine detection with CRISPR-Cas13a/C2c2. Chem. Sci. 10, 2975-2979. doi: 10.1039/C8SC03408G

Cox, D. B. T., Gootenberg, J. S., Abudayyeh, O. O., Franklin, B., Kellner, M. J., Joung, J., et al. (2017). RNA editing with CRISPR-Cas13. Science 358, 1019-1027. doi: 10.1126/science.aaq0180

Dong, D., Ren, K., Qiu, X., Zheng, J., Guo, M., Guan, X., et al. (2016). The crystal structure of Cpf1 in complex with CRISPR RNA. Nature 532, 522-526. doi: $10.1038 /$ nature 17944

Endo, A., and Toki, S. (2019). Targeted mutagenesis using FnCpf1 in tobacco. Methods Mol. Biol. 1917, 269-281. doi: 10.1007/978-1-4939-8991-1_20 suggestions. DZ designed the review, reviewed it, and approved the submitted manuscript.

\section{FUNDING}

This study was funded by the National Key R\&D Program of China under Grant No. 2018YFA0900300, the National Natural Science Foundation of China under Grant Nos. 31670604, 31970324, and 31800086, the Natural Science Foundation of Liaoning Province of China under Grant No. 2019020758, the Tianjin Synthetic Biotechnology Innovation Capacity Improvement Project under Grant No. TSBICIP-KJGG004-03, the Tianjin Science Fund for Distinguished Young Scholars under Grant No. 17JCJQJC45300, and the Science and Technology Project of Education Department of Liaoning Province under Grant No. 819001110761.

Fagerlund, R. D., Staals, R. H., and Fineran, P. C. (2015). The Cpf1 CRISPR-Cas protein expands genome-editing tools. Genome Biol. 16:251. doi: 10.1186/s13059-015-0824-9

Faure, G., Shmakov, S. A., Yan, W. X., Cheng, D. R., Scott, D. A., Peters, J. E., et al. (2019). CRISPR-Cas in mobile genetic elements: counter-defence and beyond. Nat. Rev. Microbiol. 17, 513-525. doi: 10.1038/s41579-0190204-7

Ferenczi, A., Pyott, D. E., Xipnitou, A., and Molnar, A. (2017). Efficient targeted DNA editing and replacement in Chlamydomonas reinhardtii using Cpf1 ribonucleoproteins and single-stranded DNA. Proc. Natl. Acad. Sci. U.S.A. 114, 13567-13572. doi: 10.1073/pnas.1710597114

Fernandez, J. P., Vejnar, C. E., Giraldez, A. J., Rouet, R., and Moreno-Mateos, M. A. (2018). Optimized CRISPR-Cpf1 system for genome editing in zebrafish. Methods 150, 11-18. doi: 10.1016/j.ymeth.2018.06.014

Fonfara, I., Richter, H., Bratovic, M., Le Rhun, A., and Charpentier, E. (2016). The CRISPR-associated DNA-cleaving enzyme Cpf1 also processes precursor CRISPR RNA. Nature 532, 517-521. doi: 10.1038/nature17945

Gao, P., Yang, H., Rajashankar, K. R., Huang, Z., and Patel, D. J. (2016). Type V CRISPR-Cas Cpfl endonuclease employs a unique mechanism for crRNA-mediated target DNA recognition. Cell Res. 26, 901-913. doi: $10.1038 / \mathrm{cr} .2016 .88$

Gootenberg, J. S., Abudayyeh, O. O., Lee, J. W., Essletzbichler, P., Dy, A. J., Joung, J., et al. (2017). Nucleic acid detection with CRISPR-Cas13a/C2c2. Science 356, 438-442. doi: 10.1126/science.aam9321

Hajizadeh Dastjerdi, A., Newman, A., and Burgio, G. (2019). The expanding class 2 CRISPR toolbox: diversity, applicability, and targeting drawbacks. BioDrugs 33, 503-513. doi: 10.1007/s40259-019-00369-y

Harrington, L. B., Burstein, D., Chen, J. S., Paez-Espino, D., Ma, E., Witte, I. P., et al. (2018). Programmed DNA destruction by miniature CRISPR-Cas14 enzymes. Science 362, 839-842. doi: 10.1126/science.aav4294

Harrington, L. B., Ma, E., Chen, J. S., Witte, I. P., Gertz, D., Paez-Espino, D., et al. (2020). A scoutRNA is required for some type V CRISPR-Cas systems. Mol. Cell 79, 416-424 e415. doi: 10.1016/j.molcel.2020.06.022

Hew, B. E., Sato, R., Mauro, D., Stoytchev, I., and Owens, J. B. (2019). RNA-guided piggyBac transposition in human cells. Synth. Biol. 4:ysz018. doi: 10.1093/synbio/ysz018

Hong, W., Zhang, J., Cui, G., Wang, L., and Wang, Y. (2018). Multiplexed CRISPR-Cpf1-mediated genome editing in Clostridium difficile toward the understanding of pathogenesis of C. difficile infection. ACS Synth. Biol. 7, 1588-1600. doi: 10.1021/acssynbio.8b00087

Jeon, Y., Choi, Y. H., Jang, Y., Yu, J., Goo, J., Lee, G., et al. (2018). Direct observation of DNA target searching and cleavage by CRISPR-Cas12a. Nat. Commun. 9:2777. doi: 10.1038/s41467-018-05245-x

Jia, H., Orbovic, V., and Wang, N. (2019). CRISPR-LbCas12amediated modification of citrus. Plant Biotechnol. J. 17, 1928-1937. doi: $10.1111 /$ pbi. 13109 
Jiang, Y., Qian, F., Yang, J., Liu, Y., Dong, F., Xu, C., et al. (2017). CRISPR-Cpf1 assisted genome editing of Corynebacterium glutamicum. Nat. Commun. 8:15179. doi: 10.1038/ncomms1 5179

Jiménez, A., Hoff, B., and Revuelta, J. L. (2020). Multiplex genome editing in Ashbya gossypii using CRISPR-Cpf1. N. Biotechnol. 57, 29-33. doi: $10.1016 /$ j.nbt.2020.02.002

Jinek, M., Jiang, F., Taylor, D. W., Sternberg, S. H., Kaya, E., Ma, E., et al. (2014). Structures of Cas9 endonucleases reveal RNA-mediated conformational activation. Science 343:1247997. doi: 10.1126/science.1247997

Kim, D., Kim, J., Hur, J. K., Been, K. W., Yoon, S. H., and Kim, J. S. (2016). Genome-wide analysis reveals specificities of Cpf1 endonucleases in human cells. Nat. Biotechnol. 34, 863-868. doi: 10.1038/nbt.3609

Kim, S. K., Kim, H., Ahn, W. C., Park, K. H., Woo, E. J., Lee, D. H., et al. (2017). Efficient transcriptional gene repression by type V-A CRISPR-Cpf1 from Eubacterium eligens. ACS Synth. Biol. 6, 1273-1282. doi: 10.1021/acssynbio.6b00368

Kim, Y., Cheong, S. A., Lee, J. G., Lee, S. W., Lee, M. S., Baek, I. J., et al. (2016). Generation of knockout mice by Cpf1-mediated gene targeting. Nat. Biotechnol. 34, 808-810. doi: $10.1038 /$ nbt.3614

Koonin, E. V., Makarova, K. S., and Wolf, Y. I. (2017). Evolutionary genomics of defense systems in archaea and bacteria. Annu. Rev. Microbiol. 71, 233-261. doi: 10.1146/annurev-micro-090816-093830

Kovač, A., Miskey, C., Menzel, M., Grueso, E., Gogol-Döring, A., and Ivics, Z. (2020). RNA-guided retargeting of Sleeping Beauty transposition in human cells. Elife 9:e53868. doi: 10.7554/eLife.53868.sa2

Li, B., Rui, H., Li, Y., Wang, Q., Alariqi, M., Qin, L., et al. (2019). Robust CRISPR/Cpf1 (Cas12a)-mediated genome editing in allotetraploid cotton (Gossypium hirsutum). Plant Biotechnol. J. 17, 1862-1864. doi: $10.1111 /$ pbi.13147

Li, L., Wei, K., Zheng, G., Liu, X., Chen, S., Jiang, W., et al. (2018). CRISPR-Cpf1-assisted multiplex genome editing and transcriptional repression in streptomyces. Appl. Environ. Microbiol. 84:e00827-18 doi: 10.1128/AEM.00827-18

Li, S. Y., Cheng, Q. X., Liu, J. K., Nie, X. Q., Zhao, G. P., and Wang, J. (2018a). CRISPR-Cas12a has both cis- and trans-cleavage activities on single-stranded DNA. Cell Res. 28, 491-493. doi: 10.1038/s41422-018-0022-X

Li, S. Y., Cheng, Q. X., Wang, J. M., Li, X. Y., Zhang, Z. L., Gao, S., et al. (2018b). CRISPR-Cas12a-assisted nucleic acid detection. Cell Discov. 4: 20. doi: 10.1038/s41421-019-0083-0

Li, X., Wang, Y., Liu, Y., Yang, B., Wang, X., Wei, J., et al. (2018). Base editing with a Cpf1-cytidine deaminase fusion. Nat. Biotechnol. 36, 324-327. doi: $10.1038 /$ nbt.4102

Li, Z. H., Liu, M., Wang, F. Q., and Wei, D. Z. (2018). Cpf1-assisted efficient genomic integration of in vivo assembled DNA parts in Saccharomyces cerevisiae. Biotechnol. Lett. 40, 1253-1261. doi: 10.1007/s10529-0182574-8

Liang, M., Li, Z., Wang, W., Liu, J., Liu, L., Zhu, G., et al. (2019). A CRISPR-Cas12aderived biosensing platform for the highly sensitive detection of diverse small molecules. Nat. Commun. 10:3672. doi: 10.1038/s41467-019-11648-1

Liu, J. J., Orlova, N., Oakes, B. L., Ma, E., Spinner, H. B., Baney, K. L. M., et al. (2019). CasX enzymes comprise a distinct family of RNA-guided genome editors. Nature 566, 218-223. doi: 10.1038/s41586-019-0908-X

Liu, L., Li, X., Wang, J., Wang, M., Chen, P., Yin, M., et al. (2017). Two distant catalytic sites are responsible for C2c2 RNase activities. Cell 168, 121-134.e112. doi: 10.1016/j.cell.2016.12.031

Liu, W., Tang, D., Wang, H., Lian, J., Huang, L., and Xu, Z. (2019). Combined genome editing and transcriptional repression for metabolic pathway engineering in Corynebacterium glutamicum using a catalytically active Cas12a. Appl. Microbiol. Biotechnol. 103, 8911-8922. doi: 10.1007/s00253-019-10118-4

Ma, E., Harrington, L. B., O'Connell, M. R., Zhou, K., and Doudna, J. A. (2015). Single-stranded DNA cleavage by divergent CRISPR-Cas9 enzymes. Mol. Cell 60, 398-407. doi: 10.1016/j.molcel.2015.10.030

Makarova, K. S., Wolf, Y. I., Alkhnbashi, O. S., Costa, F., Shah, S. A., Saunders, S. J., et al. (2015). An updated evolutionary classification of CRISPR-Cas systems. Nat. Rev. Microbiol. 13, 722-736. doi: 10.1038/nrmicro3569

Malzahn, A. A., Tang, X., Lee, K., Ren, Q., Sretenovic, S., Zhang, Y., et al. (2019). Application of CRISPR-Cas12a temperature sensitivity for improved genome editing in rice, maize, and Arabidopsis. BMC Biol. 17:9. doi: 10.1186/s12915-019-0629-5

Martella, A., Firth, M., Taylor, B. J. M., Göppert, A., Cuomo, E. M., Roth, R. G., et al. (2019). Systematic evaluation of CRISPRa and CRISPRi modalities enables development of a multiplexed, orthogonal gene activation and repression system. ACS Synth. Biol. 8, 1998-2006. doi: 10.1021/acssynbio.8b0 0527

McMahon, M. A., and Rahdar, M. (2021). Gene disruption using chemically modified CRISPR-Cpf1 RNA. Methods Mol. Biol. 2162, 49-60. doi: 10.1007/978-1-0716-0687-2_4

Ming, M., Ren, Q., Pan, C., He, Y., Zhang, Y., Liu, S., et al. (2020). CRISPRCas12b enables efficient plant genome engineering. Nat. Plants 6, 202-208. doi: 10.1038/s41477-020-0614-6

Moreno-Mateos, M. A., Fernandez, J. P., Rouet, R., Vejnar, C. E., Lane, M. A., Mis, E., et al. (2017). CRISPR-Cpf1 mediates efficient homology-directed repair and temperature-controlled genome editing. Nat. Commun. 8:2024. doi: 10.1038/s41467-017-01836-2

Murugan, K., Babu, K., Sundaresan, R., Rajan, R., and Sashital, D. G. (2017). The revolution continues: newly discovered systems expand the CRISPR-Cas toolkit. Mol. Cell 68, 15-25. doi: 10.1016/j.molcel.2017.09.007

Nissim, L., Perli, S. D., Fridkin, A., Perez-Pinera, P., and Lu, T. K. (2014). Multiplexed and programmable regulation of gene networks with an integrated RNA and CRISPR/Cas toolkit in human cells. Mol. Cell 54, 698-710. doi: 10.1016/j.molcel.2014.04.022

Niu, T. C., Lin, G. M., Xie, L. R., Wang, Z. Q., Xing, W. Y., Zhang, J. Y., et al. (2019). Expanding the potential of CRISPR-Cpf1-based genome editing technology in the cyanobacterium anabaena PCC 7120. ACS Synth. Biol. 8, 170-180. doi: 10.1021/acssynbio.8b00437

Nunez, J. K., Lee, A. S., Engelman, A., and Doudna, J. A. (2015). Integrasemediated spacer acquisition during CRISPR-Cas adaptive immunity. Nature 519, 193-198. doi: 10.1038/nature14237

Pausch, P., Al-Shayeb, B., Bisom-Rapp, E., Tsuchida, C. A., Li, Z., Cress, B. F., et al. (2020). CRISPR-Cas $\Phi$ from huge phages is a hypercompact genome editor. Science 369, 333-337. doi: 10.1126/science.abb1400

Pawelczak, K. S., Gavande, N. S. P. S., VanderVere-Carozza, and Turchi, J. J. (2018). Modulating DNA repair pathways to improve precision genome engineering. ACS Chem. Biol. 13, 389-396. doi: 10.1021/acschembio.7b00777

Peters, J. E. (2014). Tn7. Microbiol. Spectr. 2:MDNA30010-2014. doi: 10.1128/microbiolspec.MDNA3-0010-2014

Port, F., and Bullock, S. L. (2016). Augmenting CRISPR applications in Drosophila with tRNA-flanked sgRNAs. Nat. Methods 13, 852-854. doi: $10.1038 /$ nmeth.3972

Ramesh, A., Ong, T., Garcia, J. A., Adams, J., and Wheeldon, I. (2020). Guide RNA engineering enables dual purpose CRISPR-Cpf1 for simultaneous gene editing and gene regulation in Yarrowia lipolytica. ACS Synth. Biol. 9, 967-971. doi: 10.1021/acssynbio.9b00498

Ran, F. A., Hsu, P. D., Lin, C. Y., Gootenberg, J. S., Konermann, S., Trevino, A. E., et al. (2013a). Double nicking by RNA-guided CRISPR Cas9 for enhanced genome editing specificity. Cell 154, 1380-1389. doi: 10.1016/j.cell.2013.08.021

Ran, F. A., Hsu, P. D., Wright, J., Agarwala, V., Scott, D. A., and Zhang, F. (2013b). Genome engineering using the CRISPR-Cas9 system. Nat. Protoc. 8 , 2281-2308. doi: 10.1038/nprot.2013.143

Sakuma, T., Nishikawa, A., Kume, S., Chayama, K., and Yamamoto, T. (2014). Multiplex genome engineering in human cells using all-in-one CRISPR/Cas9 vector system. Sci. Rep. 4:5400. doi: 10.1038/srep05400

Savage, D. F. (2019). Cas14: big advances from small CRISPR proteins. Biochemistry 58, 1024-1025. doi: 10.1021/acs.biochem.9b00035

Schunder, E., Rydzewski, K., Grunow, R., and Heuner, K. (2013). First indication for a functional CRISPR/Cas system in Francisella tularensis. Int. J. Med. Microbiol. 303, 51-60. doi: 10.1016/j.ijmm.2012.11.004

Shen, W., Zhang, J., Geng, B., Qiu, M., Hu, M., Yang, Q., et al. (2019). Establishment and application of a CRISPR-Cas12a assisted genomeediting system in Zymomonas mobilis. Microb. Cell Fact 18:162. doi: 10.1186/s12934-019-1219-5

Shmakov, S., Abudayyeh, O. O., Makarova, K. S., Wolf, Y. I., Gootenberg, J. S., Semenova, E., et al. (2015). Discovery and functional characterization of diverse class 2 CRISPR-Cas systems. Mol. Cell 60, 385-397. doi: 10.1016/j.molcel.2015.10.008 
Shmakov, S., Smargon, A., Scott, D., Cox, D., Pyzocha, N., Yan, W., et al. (2017). Diversity and evolution of class 2 CRISPR-Cas systems. Nat. Rev. Microbiol. 15, 169-182. doi: 10.1038/nrmicro.2016.184

Skelding, Z., Queen-Baker, J., and Craig, N. L. (2003). Alternative interactions between the $\operatorname{Tn} 7$ transposase and the Tn7 target DNA binding protein regulate target immunity and transposition. Embo J. 22, 5904-5917. doi: 10.1093/emboj/cdg551

Sorek, R., Lawrence, C. M., and Wiedenheft, B. (2013). CRISPR-mediated adaptive immune systems in bacteria and archaea. Annu. Rev. Biochem. 82, 237-266. doi: 10.1146/annurev-biochem-072911-172315

Standage-Beier, K., Zhang, Q., and Wang, X. (2015). Targeted large-scale deletion of bacterial genomes using CRISPR-nickases. ACS Synth. Biol. 4, 1217-1225. doi: 10.1021/acssynbio.5b00132

Stella, S., Alcon, P., and Montoya, G. (2017). Structure of the Cpf1 endonuclease R-loop complex after target DNA cleavage. Nature 546, 559-563. doi: 10.1038 /nature22398

Stellwagen, A. E., and Craig, N. L. (1997). Avoiding self: two Tn7-encoded proteins mediate target immunity in Tn7 transposition. Embo J. 16, 6823-6834. doi: $10.1093 / \mathrm{emboj} / 16.22 .6823$

Sternberg, S. H., LaFrance, B., Kaplan, M., and Doudna, J. A. (2015). Conformational control of DNA target cleavage by CRISPR-Cas9. Nature 527, 110-113. doi: 10.1038/nature15544

Strecker, J., Jones, S., Koopal, B., Schmid-Burgk, J., Zetsche, B., Gao, L., et al. (2019a). Engineering of CRISPR-Cas12b for human genome editing. Nat. Commun. 10:212. doi: 10.1038/s41467-018-08224-4

Strecker, J., Ladha, A., Gardner, Z., Schmid-Burgk, J. L., Makarova, K. S., Koonin, E. V., et al. (2019b). RNA-guided DNA insertion with CRISPR-associated transposases. Science 365, 48-53. doi: 10.1126/science.aax9181

Sun, B., Yang, J., Yang, S., Ye, R. D., Chen, D., and Jiang, Y. (2018). A CRISPR-Cpf1-assisted non-homologous end joining genome editing system of Mycobacterium smegmatis. Biotechnol. J. 13:e1700588. doi: 10.1002/biot.201700588

Sun, J., Wang, Q., Jiang, Y., Wen, Z., Yang, L., Wu, J., et al. (2018). Genome editing and transcriptional repression in Pseudomonas putida KT2440 via the type II CRISPR system. Microb. Cell Fact 17:41. doi: 10.1186/s12934-018-0887-x

Swarts, D. C., and Jinek, M. (2019). Mechanistic Insights into the cisand trans-acting DNase activities of Cas12a. Mol. Cell 73, 589-600 e584. doi: 10.1016/j.molcel.2018.11.021

Teng, F., Cui, T., Feng, G., Guo, L., Xu, K., Gao, Q., et al. (2018). Repurposing CRISPR-Cas $12 \mathrm{~b}$ for mammalian genome engineering. Cell Discov. 4:63. doi: 10.1038/s41421-018-0069-3

Teng, F., Guo, L., Cui, T., Wang, X. G., Xu, K., Gao, Q., et al. (2019a). CDetection: CRISPR-Cas12b-based DNA detection with sub-attomolar sensitivity and single-base specificity. Genome Biol. 20:132. doi: 10.1186/s13059-0191742-z

Teng, F., Li, J., Cui, T., Xu, K., Guo, L., Gao, Q., et al. (2019b). Enhanced mammalian genome editing by new Cas12a orthologs with optimized crRNA scaffolds. Genome Biol. 20:15. doi: 10.1186/s13059-019-1620-8

Ungerer, J., and Pakrasi, H. B. (2016). Cpf1 is a versatile tool for CRISPR genome editing across diverse species of cyanobacteria. Sci. Rep. 6:39681. doi: $10.1038 /$ srep39681

Vanegas, K. G., Jarczynska, Z. D., Strucko, T., and Mortensen, U. H. (2019). Cpf1 enables fast and efficient genome editing in Aspergilli. Fungal Biol. Biotechnol. 6:6. doi: 10.1186/s40694-019-0069-6

Vu, T. V., Sivankalyani, V., Kim, E. J., Doan, D. T. H., Tran, M. T., Kim, J., et al. (2020). Highly efficient homology-directed repair using CRISPR/Cpf1-geminiviral replicon in tomato. Plant Biotechnol. J. 18:2133-2143. doi: 10.1111/pbi.13373

Wang, X., Ding, C., Yu, W., Wang, Y., He, S., Yang, B., et al. (2020a). Cas12a base editors induce efficient and specific editing with low DNA damage response. Cell Rep. 31:107723. doi: 10.1016/j.celrep.2020.107723

Wang, X., Zhong, M., Liu, Y., Ma, P., Dang, L., Meng, Q., et al. (2020b). Rapid and sensitive detection of COVID-19 using CRISPR/Cas12a-based detection with naked eye readout, CRISPR/Cas12a-NE. Sci. Bull. 65, 1436-1439. doi: 10.1016/j.scib.2020.04.041

Wu, D., Guan, X., Zhu, Y., Ren, K., and Huang, Z. (2017). Structural basis of stringent PAM recognition by CRISPR-C2c1 in complex with sgRNA. Cell Res. 27, 705-708. doi: 10.1038/cr.2017.46

Wu, F., Qiao, X., Zhao, Y., Zhang, Z., Gao, Y., Shi, L., et al. (2020). Targeted mutagenesis in Arabidopsis thaliana using CRISPR-Cas12b/C2c1. J. Integr. Plant Biol. 62, 1653-1658. doi: 10.1111/jipb.12944

Wu, Y., Liu, Y., Lv, X., Li, J., Du, G., and Liu, L. (2020). CAMERS-B: CRISPR/Cpf1 assisted multiple-genes editing and regulation system for Bacillus subtilis. Biotechnol. Bioeng. 117, 1817-1825. doi: 10.1002/bit.27322

Xu, T., Li, Y., Shi, Z., Hemme, C. L., Li, Y., Zhu, Y., et al. (2015). Efficient genome editing in Clostridium cellulolyticum via CRISPR-Cas9 nickase. Appl. Environ. Microbiol. 81, 4423-4431. doi: 10.1128/AEM.00873-15

Yan, W. X., Hunnewell, P., Alfonse, L. E., Carte, J. M., Keston-Smith, E., Sothiselvam, S., et al. (2019). Functionally diverse type V CRISPR-Cas systems. Science 363, 88-91. doi: 10.1126/science.aav7271

Yang, H., Gao, P., Rajashankar, K. R., and Patel, D. J. (2016). PAM-dependent target DNA recognition and cleavage by C2c1 CRISPR-Cas endonuclease. Cell 167, 1814-1828 e1812. doi: 10.1016/j.cell.2016.11.053

Yang, Z., Edwards, H., and Xu, P. (2020). CRISPR-Cas12a/Cpf1-assisted precise, efficient and multiplexed genome-editing in Yarrowia lipolytica. Metab. Eng. Commun. 10:e00112. doi: 10.1016/j.mec.2019.e00112

Yin, X., Anand, A., Quick, P., and Bandyopadhyay, A. (2019). Editing a Stomatal Developmental Gene in Rice with CRISPR/Cpf1. Methods Mol Biol 1917: 257-268. doi: 10.1007/978-1-4939-8991-1_19

Yosef, I., Goren, M. G., and Qimron, U. (2012). Proteins and DNA elements essential for the CRISPR adaptation process in Escherichia coli. Nucleic Acids Res. 40, 5569-5576. doi: 10.1093/nar/gks216

Zetsche, B., Gootenberg, J. S., Abudayyeh, O. O., Slaymaker, I. M., Makarova, K. S., Essletzbichler, P., et al. (2015). Cpf1 is a single RNA-guided endonuclease of a class 2 CRISPR-Cas system. Cell 163, 759-771. doi: 10.1016/j.cell.2015.09.038

Zetsche, B., Heidenreich, M., Mohanraju, P., Fedorova, I., Kneppers, J., DeGennaro, E. M., et al. (2017). Multiplex gene editing by CRISPR-Cpf1 using a single crRNA array. Nat. Biotechnol. 35, 31-34. doi: 10.1038/nbt.3737

Zhang, J., Hong, W., Zong, W., Wang, P., and Wang, Y. (2018). Markerless genome editing in Clostridium beijerinckii using the CRISPR-Cpf1 system. J. Biotechnol. 284, 27-30. doi: 10.1016/j.jbiotec.2018.07.040

Zhang, J., Yang, F., Yang, Y., Jiang, Y., and Huo, Y. X. (2019). Optimizing a CRISPR-Cpf1-based genome engineering system for Corynebacterium glutamicum. Microb. Cell Fact 18:60. doi: 10.1186/s12934-019-1109-x

Zhang, J. L., Peng, Y. Z., Liu, D., Liu, H., Cao, Y. X., Li, B. Z., et al. (2018). Gene repression via multiplex gRNA strategy in Y. lipolytica. Microb. Cell Fact 17:62. doi: 10.1186/s12934-018-0909-8

Zhang, X., Wang, J., Cheng, Q., Zheng, X., Zhao, G., and Wang, J. (2017). Multiplex gene regulation by CRISPR-ddCpf1. Cell Discov. 3:17018. doi: 10.1038/celldisc.2017.18

Zou, Y.-l., Li, Z.-y., Zou, Y.-j., Hao, H.-y., Hu, J.-x., Li, N., et al. (2019). Generation of pigs with a Belgian Blue mutation in MSTN using CRISPR/Cpf1-assisted ssODN-mediated homologous recombination. J. Integr. Agric. 18, 1329-1336. doi: 10.1016/S2095-3119(19)62694-8

Conflict of Interest: The authors declare that the research was conducted in the absence of any commercial or financial relationships that could be construed as a potential conflict of interest.

Copyright (C) 2021 Tong, Dong, Cui, Jiang, Jin and Zhang. This is an open-access article distributed under the terms of the Creative Commons Attribution License (CC $B Y)$. The use, distribution or reproduction in other forums is permitted, provided the original author(s) and the copyright owner(s) are credited and that the original publication in this journal is cited, in accordance with accepted academic practice. No use, distribution or reproduction is permitted which does not comply with these terms. 